\title{
Presentación Dossier: Transporte, movilidad y territorio: perspectivas a partir de la pandemia COVID-19
}

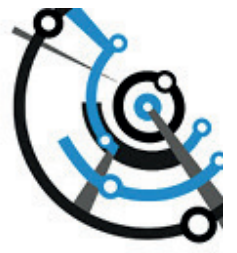

( Andrea Gutiérrez

Universidad de Buenos Aires, Facultad de Filosofía y Letras, Instituto de Geografía, Programa Transporte y Territorio; CONICET, Argentina.

ORCID: https://orcid.org/oooo-ooo2-8124-1679

\section{Jorge Blanco}

Universidad de Buenos Aires, Facultad de Filosofía y Letras, Instituto de Geografía, Programa Transporte y Territorio, Argentina.

ORCID: https://orcid.org/oooo-0002-2258-4540

La pandemia COVID-19 es un evento de alta significación en la historia reciente de la humanidad, que habilita una reflexión profunda en distintos campos de la producción académica y de las políticas públicas. El transporte y la movilidad constituyen un ámbito de interés central, interpelado y desafiado por las condiciones inéditas que se generaron a partir de los primeros meses de 2020 en todo el mundo. La intensidad, diversidad, desigualdad, multidireccionalidad, multiescalaridad y velocidad de los flujos son algunos de los rasgos característicos de las sociedades contemporáneas, que pueden ser vistos tanto desde su papel constitutivo en la generación y difusión de la pandemia, como en los efectos que el procesamiento de ésta ha generado sobre esas condiciones de circulación.

La pandemia es, por su propia definición, territorial y su evolución ha mostrado que la clave territorial es básica para su comprensión y también para imaginar nuevos caminos para el futuro próximo del transporte y de la movilidad. Cuestiones tales como el alcance global de la pandemia, su rápida difusión a través de sujetos y redes de circulación, o la impactante detención de un mundo en constante movimiento, abren la posibilidad de volver a examinar el papel de la circulación en el mundo actual. Asimismo, el transporte y la movilidad están íntimamente asociados con distintas políticas y acciones por parte de los diferentes niveles de gobierno dirigidas a regular el confinamiento de ciudades, regiones y países; a establecer restricciones a la circulación, diferenciando sectores esenciales y críticos; a normar sobre el uso del transporte público y el privado, promoviendo nuevas formas de desplazamiento, modificando operatorias y rutinas del transporte; a alertar sobre la necesidad de desarrollar nuevas prácticas y comportamientos en los distintos momentos que componen un viaje.

Los procesos de conexión digital han ocupado un lugar aún más central en la sociedad, y han profundizado sus articulaciones con la circulación material que incluyen sustituciones, sinergias, desarrollos conjuntos, al tiempo que se modifican las formas de 
trabajar, producir y distribuir mercaderías, y se generan nuevas formas de sociabilidad. El transporte, la movilidad y la información también encarnan una tríada vinculada con las prácticas sociales en tiempos de pandemia.

Las ciudades fueron uno de los escenarios más visibles de los impactos de la expansión del COVID-19, tanto por su rol en la difusión de la pandemia como por las imágenes inéditas que ofrecieron frente a las medidas de cuidado sanitario y aislamiento social, con el consiguiente repliegue hacia el interior de los hogares. Las redes de circulación a distintas escalas sirvieron de soporte para la difusión de la pandemia, con particular atención a las regiones metropolitanas y grandes ciudades que actuaron tanto como puertas de entrada del virus como de ámbito privilegiado para su posterior expansión. Se combinaron así un proceso de difusión propio de la globalización a través de las redes multiescalares, con los procesos tradicionales de difusión en el marco de las redes urbanas -a saltos- y de difusión por contigüidad, particularmente crítico hacia el interior de las regiones metropolitanas funcionalmente articuladas.

En cuanto a las imágenes urbanas, fuimos testigos de la desolación en áreas centrales y calles, que habitualmente exhibían los rasgos de la intensa movilidad que caracteriza a la sociedad contemporánea. Los termómetros de la movilidad fueron indicando, a través de los registros tecnológicos, la casi total detención de los movimientos urbanos o su abrupta reducción, restringidos a la proximidad y a lo esencial.

Diversas acciones se han desarrollado en relación con el transporte y la movilidad en esta encrucijada crítica. Estas acciones estaban atravesadas por los protocolos de resguardo de la salud, por la necesidad de mantenimiento de una cierta oferta básica de servicios, por recomendaciones para modificar elecciones modales, por restricciones espacio-temporales para la movilidad. Se trata de una encrucijada compleja que requiere acciones en el corto plazo y en el marco de gran incertidumbre, pero que puede volverse un terreno fértil para recuperar las agendas pendientes incompletas -que se volvieron aún más evidentes-, revisar los escenarios tendenciales e imaginar nuevos futuros. El dossier propone una exploración de este amplio espectro de temas abriendo nuevas perspectivas sobre el transporte, la movilidad y el territorio en un tiempo de incertidumbres y riesgos, e invita a generar propuestas reflexivas a partir de lo que fue, lo que está siendo y lo que probablemente será y emerge como agenda.

Las singulares características de este dossier, de producción sincrónica al proceso abordado, conllevan un significativo esfuerzo de identificación, seguimiento y sistematización de estudios e información de fuentes diversas, necesarios para componer el panorama sobre el cual reflexionar, con un estilo predominantemente ensayístico. Por su intermedio, los artículos ofrecen un estado de la cuestión cuya captura es, en sí misma, un aporte valioso. Cabe puntualizar que, conforme a los tiempos del proceso editorial, los trabajos de este dossier fueron elaborados durante 2020 y hasta comienzos de 2021, en pleno curso de la pandemia y aun sin vislumbrarse los efectos de la vacunación más masiva y a escala ampliada en lo internacional.

El dossier se compone de siete artículos que abordan algunos de estos múltiples ángulos desde los cuales pueden pensarse la movilidad, el transporte y el territorio en tiempos de pandemia y postpandemia. Presentan un cuadro variado, selectivo y necesariamente provisorio, algo inevitable en tiempos que se definen por la incertidumbre y lo imprevisto. Los trabajos abordan cuestiones vinculadas con las tecnologías de la información y comunicación (TICs), la logística (tanto en redes globales como en contextos urbanos), la gobernanza y las políticas públicas, aspectos parciales e integrales de las movilidades y propuestas de nuevas perspectivas para pensar la tríada con los transportes y el territorio. No obstante esta diversidad, sus revisiones y análisis comparten una estructura similar: aportan un contexto acerca 
de la situación precedente, identifican impactos -tanto negativos como ventajas o avances-, y estrategias y capacidades de resiliencia (de preparación, respuesta y recuperación frente a acontecimientos disruptivos).

En ese conjunto diverso, algunos temas emergen a través de la secuencia de artículos. La sustentabilidad es un rasgo y una condición de las movilidades posibles con la que dialogan los textos de Brasileiro et al. y de Roig-Costa et al. En particular es "la sustentabilidad y la(s) crisis pandémicas y ambientales" uno de los ejes de esos trabajos que, sin embargo, se encuentran entre los extremos de una mirada macro y una mirada micro, lecturas ambas habilitadas por la propia complejidad de la situación. Sustentabilidad no sólo en términos ambientales sino también económicos, institucionales y sociales, así como también señalan los trabajos de Pulga et al., Borthagaray y Gutiérrez, y Figueroa y Gurdon, en relación con el transporte público y con la desigualdad socioespacial de las ciudades latinoamericanas, que expresan situaciones críticas preexistentes.

Otro tema emergente es el de las TICs y la logística, rastreable a través de los trabajos de Martner, Pulga et al., y Borthagaray y Gutiérrez. El caleidoscopio gira en un juego de escalas y en la transversalidad de estos componentes en las movilidades globales y urbanas. La 4ta. revolución industrial se muestra como un proceso de maduración de la 3ra., aún en desarrollo, con efectos pendulares en cuanto a sus atributos en relación con el espacio-tiempo. Así como la economía y el territorio de redes y flujos (materiales y de información) transformó al capitalismo mediante la fragmentación espacial funcionalmente integrada de actividades y llevó a la globalización de las cadenas de producción y distribución sustentadas en ventajas competitivas de precios con fluidez y confiablidad, la pandemia evidenció vulnerabilidades ante eventos de cambio, y puso en cuestión a la "lejanía" (y también a la proximidad).

Una profundización de la transición digital se vislumbra en la proyección a futuro de la 4ta. revolución, con un papel preponderante de la gestión numérica hacia la digitalización de procesos, la trazabilidad, la información para imprevistos y para planes y acciones de contingencia flexibles. También se vislumbran efectos desiguales: mayor encarecimiento de fletes marítimos e insuficiencia de inventarios en los países no centrales de la economía mundo, mayor uso de los aplicativos en relación con el poder adquisitivo, mayor accesibilidad a ventajas laborales y económicas sostenidas en la generación de empleo precario sin garantías de trabajo.

Los modelos urbanos y de movilidad están en el centro de varios artículos, tanto para pensar la diversidad de estrategias adoptadas en el contexto de la pandemia (Borthagaray y Gutiérrez), como para evaluar las experiencias de micromovilidad (Roig-Costa et al.) y, más aún, para pensar en la ciudad y la movilidad desde nuevas perspectivas, en las que se resignifican el rol del Estado y de la sociedad en la gestión de los servicios urbanos (Brasileiro et al., Andrade et al.). Entre los distintos autores se manifiesta un acuerdo en cuanto a la mantención de tendencias vinculadas con ciertos motivos de viajes y medios de transporte, en particular con las compras en línea, los viajes de negocios y viajes pendulares por trabajo, una función presencial intermitente en educación (especialmente en la superior) y el uso de opciones de movilidad activa (bicicleta o caminata principalmente). Constituyen alternativas reales con sustento en el potencial de las TICS para cambios que tornarán crecientemente importante a la generación de datos y de diagnósticos.

Finalmente, todos los artículos navegan entre las agendas consensuadas en torno a lineamientos básicos de políticas de movilidad y las tendencias observables en la situación de pandemia. Con respecto a este particular también se manifiesta en los distintos autores una remisión -más o menos expresa- al papel del Estado, acompañada de una colocación en la agenda del campo de estudio sobre transporte y movilidad de 
políticas nacionales -públicas y empresariales- que prioricen y formulen una estructura para emergencias y gestión de desastres, frente a su creciente diversidad (climáticos, ambientales, sanitarios, etc.) y frecuencia, en atención a la seguridad, al abastecimiento y a otros derechos (Pulga et al., Martner, Andrade et al., Brasileiro et al., Borthagaray y Gutiérrez).

Tensiones, direcciones opuestas, fuerzas contrastantes y derivaciones imprevistas son recogidas y explicitadas en varios de los artículos integrantes del dossier "Transporte, movilidad y territorio: perspectivas a partir de la pandemia COVID-19", cuyos contenidos se detallan en los párrafos siguientes.

El texto de Carlos Martner Peyrelongue describe la interrupción de las cadenas globales de suministro a causa de la pandemia y analiza la reorganización logística y espacial asociada al transporte marítimo, a los puertos y a los corredores multimodales terrestres. Un elemento que atraviesa al trabajo es la reflexión en torno a la vulnerabilidad de estas cadenas distintivas y constitutivas de la economía de redes en la globalización. Si bien se identifica el acontecimiento coyuntural que desencadena en la interrupción de las cadenas logísticas globales y sus distintos efectos, ésta vulnerabilidad se enmarca en la descripción de un contexto precedente a la pandemia, y que identifica otras vulnerabilidades. La disputa comercial entre Estados Unidos y China y la caída del movimiento comercial en 2019 recupera un aumento de medidas proteccionistas y la relocalización de eslabones del proceso de fabricación en busca de una mayor proximidad que no logra acortar las distancias con respecto a los principales mercados de destino de los productos. También un aumento en la frecuencia de eventos naturales que reducen o paralizan la actividad marítimo-portuaria aunque en lugares focalizados y por períodos cortos. La pandemia, en cambio, muestra una disrupción prolongada debida a la multilocalización de las cadenas, con cierres y reactivaciones de la actividad escalonados y a destiempo, sumados a una diversificación de los problemas por las distintas acciones gubernamentales y situaciones de contexto para reaccionar ante la crisis. Evidencia asimismo rigideces de adaptación frente a cambios bruscos en el flujo de bienes, tanto por caídas en la provisión como por aumentos en la demanda. Las restricciones al consumo de servicios (viajes, gimnasios, restaurantes, espectáculos, etc.) conllevaron un explosivo crecimiento del consumo de bienes que encontró un cuello de botella en la falta de contenedores, de espacio en los buques, camiones y vagones de ferrocarril y asimismo de personal. Las demoras en los puertos de origen y destino ponen en cuestión no sólo la fluidez del suministro sino la confiabilidad del arribo, dos pilares de la producción fragmentada de bienes articulada por cadenas intermodales de logística. En cuanto a los efectos económicos de esta vulnerabilidad, emergen dos aspectos notables: uno, la vastedad de los perjuicios hacia afuera de la actividad (transversal a las diversas ramas de la industria); y dos, los beneficios hacia adentro de la actividad. La precedente gestión de datos y concentración de las empresas navieras, permitió manejar cierres de rutas y acuerdos (oligopólicos) que evitaron no sólo la caída sino un aumento del precio del flete. El consecuente debate sobre la relocalización y regionalización de las cadenas de suministro plantea interrogantes sobre su viabilidad. Uno interesante es el reconocimiento de factores de inercia territorial -como la generación de capacidades competitivas para producir la enorme cantidad de insumos y bienes intermedios- contrastantes con la creciente volatilidad del entorno global. Las tendencias emergentes apuntan a un refuerzo de la digitalización y a la construcción de redes resilientes, no tanto físicas sino entre actores (cargadores en tierra, países, distintos niveles y sectores de gobierno).

El trabajo de Pulga et al. dialoga de una manera muy rica con el de Martner, abordando las cadenas de suministro y distribución domiciliaria de bienes esenciales -alimentos y productos de higiene- comercializados mediante plataformas en línea, a partir de la experiencia brasilera. La disrupción de las cadenas logísticas globales (transversal 
a las actividades), el subsecuente desabastecimiento y aumento de precios conectan ambos trabajos, pero ahora con una mirada desde las empresas de plataformas y los servicios de delivery. Pulga et al. también enmarcan estos acontecimientos remitiendo al contexto nacional previo a la pandemia, y señalan procesos de desindustrialización y crisis económica no obstante los cuales -a diferencia de lo consignado por Martner- el movimiento comercial mediante las plataformas y los servicios de delivery experimenta un crecimiento. También se consigna el explosivo aumento de la demanda con la pandemia, tanto de consumidores y de bienes como de las empresas que los comercializan en línea (farmacias y supermercados en particular). Pero las empresas operadoras de plataformas para la entrega domiciliaria de compras en línea muestran una capacidad de adaptación ágil y con alto desempeño: incorporan clientes, perfeccionan métodos de pago y seguridad sanitaria, utilizan mecanismos de gobernanza en red (entrega), y logran una tendencia positiva de confianza en los aplicativos por parte de los consumidores. Los autores advierten sobre los peligros de una nueva concentración del poder económico frente a distintas situaciones: el perfil de los consumidores (siendo el uso de los aplicativos mayor con el poder adquisitivo), el de los trabajadores y sus condiciones laborales (vulnerables y precarias), y la poca transparencia y disponibilidad pública de información sobre la actividad (para diagnósticos del mercado y laborales). El manejo diferencial de los impactos de la crisis por parte de las empresas navieras gracias al acervo en gestión de datos y acuerdos oligopólicos es un antecedente a considerar. Otro aspecto convergente con Martner es connotar una conceptualización más amplia y completa de las cadenas de producción y distribución, incluyendo en este caso al consumidor final como parte de las mismas, también a los flujos inversos de devolución, y al agente del delivery como un eslabón intermediario entre el consumidor final y los comerciantes mayoristas y minoristas, con un rol central en la continuidad de la cadena de distribución y estructurador de una nueva organización del flujo de transporte y de información. Este rol, el despliegue de innovaciones logísticas y el peso adquirido en la generación de empleo sin garantías de trabajo, con mayor carga horaria semanal y menor remuneración durante 2020 que en 2019, iluminan contrastes llamativos. También el sustento de los flujos en un aumento de los "fijos", en stocks de seguridad con robotización del almacenaje y localización en distintas partes de la cadena -incluyendo la logística mayorista y minorista-, fenómeno compartido por las cadenas de suministro globales.

El texto "Movilidad urbana post pandemia: fuerzas en pugna con sentidos de sustentabilidad contrapuestos" de Borthagaray y Gutiérrez plantea un recorrido selectivo por experiencias, acciones y estrategias implementadas en distintos contextos urbanos durante la pandemia. Este repaso no es inventarial, sino que está en clave de las tensiones entre un cierto consenso en torno a algunos aspectos nodales de las políticas de movilidad urbana preexistentes y de las tendencias insinuadas en la pandemia, que se orientan en otra dirección. Este movimiento entre lo que se viene desarrollando y lo que puede desarrollarse, entre pasado reciente y futuro, permite señalar las consecuencias más negativas del modelo de movilidad urbana previo a la crisis del COVID $\mathrm{y}$, al mismo tiempo, abrir el interrogante sobre las posibilidades que se plantean para construir ciudades más equitativas y menos vulnerables ante crisis estructurales. La revisión de las acciones presentadas en el período de pandemia se organiza considerando iniciativas desde la oferta y desde el encuentro de ésta con la gestión de la demanda, involucrando intervenciones sobre la ciudad y sobre la diversidad de formas de desplazamiento en el espacio urbano. En complemento al abordaje de Figueroa y Gurdon, los autores subrayan la necesidad de un punto de vista territorial, en este caso como clave para comprender tanto las inadecuaciones y tensiones entre la dinámica urbana y la espacialidad de la oferta de transporte como las nuevas condiciones espaciotemporales derivadas de esperables cambios en las formas de organización del trabajo y de las diversas actividades de la vida social y cotidiana. Las conclusiones enfatizan las tendencias en pugna, hacia una restauración de lo anterior y hacia lo emergente, fuerzas 
que se contraponen con inercias históricas y territoriales, materiales y organizacionales. En todo caso, ante un futuro incierto los autores rescatan dos certezas: la continuidad de la vulnerabilidad socioeconómica y territorial, y la no linealidad de la proyección de ese futuro, pleno de contingencias, incertidumbres, conflictos y contradicciones, según sus propias palabras. Este puntapié dialoga con las indagaciones más profundas de Brasileiro et al. acerca de una nueva construcción política y de gestión de la agenda pública en movilidad urbana, y con las experiencias y reflexiones recuperadas por Roig-Costa et al., Andrade et al., Pulga et al., y Figueroa y Gurdon.

El artículo de Roig-Costa, Gómez-Varo, Cubells y Marquet "La movilidad post-pandemia: perfiles y usos de la micromovilidad en Barcelona" nos introduce en una mirada de detalle sobre algunos de los componentes más nuevos en el repertorio de estrategias de movilidad. El trabajo aporta los resultados de una encuesta sobre el uso de modos de transporte incluidos como parte del concepto de micromovilidad, que remite a desplazamientos de corta distancia realizados por vehículos pequeños, ligeros y cuya energía no proviene de un combustible fósil, en propiedad o compartido. Se enuncian así algunos de los rasgos de estas estrategias en materia de escala del movimiento, condiciones ambientales y tecnológicas y formas de uso de activos de movilidad que implican o no la propiedad. Así es como los autores incluyen en este grupo al patinete eléctrico, la bicicleta convencional compartida, la bicicleta eléctrica compartida y la moto eléctrica compartida. Es limitada la información disponible sobre el uso efectivo de estos modos, por lo que el ejercicio también tiene la función de proveer una primera serie de datos para el contexto de Barcelona y para recoger algunos cambios vinculados a la situación de la pandemia. Los principales resultados dan cuenta de un uso que combina diferencias por género y etarias, con un perfil de usuarios predominantemente compuesto por hombres jóvenes y ocupados, con nivel educativo relativamente elevado, que utilizan estos modos para todo un espectro de motivos de movilidad, entre los que se destaca el desplazamiento por trabajo. El cambio de modos de transporte en situación de pandemia tiene resultados llamativos porque las derivaciones hacia la micromovilidad provienen del transporte público y de las caminatas, por lo que una mirada integral pone dudas sobre la potencial contribución de la micromovilidad en cuestiones ambientales y de salud. En conjunto, la contribución brinda información sobre un elemento más de la complejidad y multiplicidad de movilidades metropolitanas y refuerza la idea de atender a esta diversidad frente a simplificaciones que fetichizan algunas formas de movilidad en particular.

El texto de Figueroa y Gurdon apunta a formular un pensamiento crítico sobre el despliegue de las TICs, haciendo foco en la dimensión territorial de los cambios promovidos por la pandemia desde el punto de vista urbano. Indagan, así, en el significado de las alteraciones en los patrones de movilidad para las relaciones y prácticas sociales que se tejen en y a través del territorio, y señalan un doble efecto de aumento e invisibilización de la desigualdad socioespacial en las ciudades. En acuerdo con Pulga et al., Figueroa y Gurdon recuperan la eficiencia y robustez demostrada por las TICs, logrando una extensión de los hábitos de uso -no sólo para el consumo de bienes sino también para el entretenimiento y la educación, además del trabajo, la atención médica y el encuentro social y familiar- acompañada de una masificación a inusitada velocidad. Convergen asimismo en identificar una nueva etapa en el desarrollo de un terciario avanzado, que atiende tanto a las grandes empresas como a las demandas domésticas de uso regular y frecuente. Con la mirada puesta en la vivienda como locus de múltiples actividades (además del consumo), los autores observan el cambio radical en la conceptualización del hogar como un "atractor" de viajes, a diferencia del movimiento acaecido con la revolución industrial, de separación de actividades (y lugares) laborales y de residencia. Mediante esta hiperconexión del hogar, sostienen que las relaciones se desenvuelven primeramente con lo remoto y no con el entorno físico ni mediadas por él, llevando a nuevas prácticas desterritorializadas, y a la vez, a una condición de vida más anclada, 
caminos ambos conducentes a una desvinculación con las diversas realidades urbanas (incluyendo al contexto inmediato) que invisibiliza dinámicas de diferenciación y segregación socioespacial, así como su intensificación durante la pandemia. Respecto a esto último, los autores advierten que la conexión virtual comienza a convertirse en un valor de la vida societal: la pandemia coloca a la densidad urbana como una amenaza y aumenta la desaglomeración, la conectividad torna menos dependiente del arraigo territorial a la provisión de servicios y disminuye la congestión, y se evidencian los menores rendimientos de las prácticas tradicionales para las empresas y los trabajadores que no requieren movilizarse, perfilando una nueva economía del territorio. $\mathrm{Al}$ igual que en Borthagaray y Gutiérrez, queda planteada una tensión entre fuerzas para la post pandemia: entre éstas para hacer perdurar las nuevas prácticas, y las que evidencian a la conectividad como tributaria de la capacidad económica así como de otras capacidades. Los autores concluyen que las TICs se revelan como un potente vector para acelerar la expansión urbana, la segregación socioespacial y el aislamiento físico, y colocan la necesidad de revisar conceptos como los de capital de movilidad, accesibilidad y reproducción social.

Andrade et al. ponen foco particular en los desafíos de la sustentabilidad del transporte público en las ciudades brasileras frente a la pandemia. También en este caso los autores aportan el contexto nacional precedente (tanto operacional como jurídico-institucional), y estos desafíos son vistos como emergentes de una crisis sectorial previa a la pandemia. El trabajo ofrece un diagnóstico de su impacto en el transporte público de un país referencial por sus enormes dimensiones demográficas y urbanas, cuya movilidad es satisfecha en forma predominante por el servicio de autotransporte público. Frente a una caída en la demanda en las más grandes ciudades iniciada en 2014 (São Paulo, Rio de Janeiro, Salvador, Belo Horizonte, Porto Alegre, Curitiba, Fortaleza, Recife y Goiânia) y con solo 11 sistemas con subsidios (para costear las gratuidades de ciertos grupos sociales), la pandemia condujo a reducciones de la oferta -50\%- en 314 ciudades y paralización de servicios en 181, con una caída de la demanda del orden del 70\% y un aumento de los costos operacionales (por las medidas de seguridad e higiene), encontrando restricciones por parte de los recursos públicos para su financiamiento y también infraestructurales para viabilizar los transportes activos en una escala amplia, en un marco de incertezas para hacer previsiones de demanda durante y post pandemia. Esta situación de emergencia acarreada por el COVID es percibida por los autores como una oportunidad para enfrentar cuestiones crónicas y promover cambios demorados tanto en la planificación como en la gestión de los transportes públicos, no buscando salvar a las empresas operadoras de una coyuntura sino reestablecer el sistema de movilidad y de transporte público urbano. Para ello proponen una metodología innovadora: crear un ámbito desde la universidad pública donde investigadores (nacionales y extranjeros), operadores, organizaciones no gubernamentales, gestores y políticos involucrados con la problemática de los transportes y la movilidad urbana exponen libremente sus ideas para perfilar una política de transporte. De este modo, el artículo trabaja con los datos relevados a partir de un fórum de discusión organizado por la Universidad Federal de Pernambuco y compuesto de ocho seminarios temáticos, cuyos resultados son analizados mediante técnicas combinadas cuali y cuantitativas. Por su intermedio, se identifican ideas fuerzas y significados, se sintetizan, estructuran y jerarquizan problemas, se registran las barreras a superar (económicas, financieras, sociales, institucionales, tecnológicas, legales y políticas) y se proponen objetivos y metas calificados en estratégicos, tácticos y operacionales. Estos resultados, validados por los participantes en dos seminarios, aportan una síntesis de los problemas del transporte público brasilero y de las acciones a emprender durante la crisis, consignando indicadores, fuentes de validación y actores involucrados (usuarios, operadores y poder público). Más allá de estos resultados directos, cabe consignar que de la metodología del trabajo se deriva también un mapa de los actores y del armazón jurídico de un proceso nacional más amplio, de institucionalización de derechos y de políticas. A este respecto, 
es sugerente el desfasaje entre la vanguardia jurídica brasilera (que incluye la sanción por ley de una política nacional de movilidad urbana y la declaración del transporte como derecho social constitucional, con propuestas de creación de un Sistema Único de Movilidad Urbana, semejante al de salud) y su "salida del papel" (en palabras de los autores), panorama conviviente con el descrito por Pulga et al. respecto al ascenso de nuevos servicios sustentados en plataformas virtuales, cuya concepción jurídica como servicios esenciales (aunque no públicos) también avanza, perfilando ambos un movimiento de cambio que es transversal a los desafíos de la movilidad en las ciudades de la actualidad. Por último, de la revisión de literatura se destaca un estudio sobre la propensión a dejar de utilizar el transporte público efectuado en Estocolmo a partir de las smartcards, y que indica la mayor y menor propensión en asociación directa con el estatus económico de las áreas, de cuyos resultados los autores recuperan la posibilidad de conducir a políticas para ajustar la oferta de transporte público conforme a las necesidades de la población, en vez de asumir una distribución homogénea de las demandas en la red.

Por último, en el artículo "A mobilidade sustentável como instituição do Comum: o desafio de sua inclusão na agenda das políticas públicas do Brasil pós-COVID”, Brasileiro, Meira, Rabay, Cavalcanti y dos Santos convocan, con la excusa de la pandemia, a incorporar una nueva perspectiva sobre la movilidad a través de una apelación potente para encarar una nueva construcción política y de gestión. El objetivo explícito del artículo es construir una narrativa que sensibilice a los decisores públicos sobre la necesidad de incluir la movilidad sustentable en las agendas públicas. La pandemia COVID-19 es invocada en unas pocas ocasiones en las que se señala: su alcance mundial, abarcando a todos pero diferencialmente; la forma en que profundizó condiciones socioambientales desiguales; los foros de debate que se habilitaron a partir de su irrupción como usinas de reflexión, y los interrogantes sobre los modos de vida pospandemia y los desafíos que implican. Estas menciones a la pandemia dan cuenta del contexto en el que se plantean las propuestas que el artículo desarrolla y que se centran en dos pilares claves. Las bases conceptuales son las nociones de commons o bienes comunes y las perspectivas económicas institucionalistas. La propuesta de considerar la ciudad, el espacio de circulación y los servicios públicos como bienes comunes está estrechamente asociada con el reconocimiento de necesidades, derechos y garantías de acceso a todos sus beneficiarios potenciales. El common es un proceso político que implica co-obligaciones, co-decisiones y co-gobierno, presentado como alternativa al proceso de desarrollo capitalista y resituando el papel del Estado en relación con los servicios y con el espacio público. De la perspectiva institucionalista, el artículo destaca la noción de instituciones como normas con distintos tipos de formalización que plantean las reglas de juego en las que las organizaciones y los grupos de interés despliegan sus relaciones de poder. Finalmente, el artículo liga estas ideas en torno a la movilidad sustentable como un bien común, profundizando en la necesidad de construir una narrativa políticamente potente para su incorporación en la agenda pública y para imaginar y practicar nuevas formas de concebir y gestionar la ciudad y los servicios públicos.

Vistos en conjunto, los textos que componen este dossier reconstruyen un panorama de la diversidad de los temas y problemas asociados con el transporte, la movilidad y el territorio, y hoy atravesados por la pandemia COVID-19. Como los artículos expresan sistemáticamente, la pandemia ha resignificado cuestiones centrales de la agenda contemporánea, al tiempo que también reorienta los caminos abiertos para el futuro. En este sentido, hay un complejo juego de tendencias que se insinúan y se potencian, en tanto ciertas posibilidades -apenas esbozadas y más lejanas en el mundo prepandemia- se constituyen en senderos más reales de un horizonte vecino y que interpela. Entendemos que los múltiples foros de debate abiertos ante esta coyuntura mundial han enriquecido particularmente a este campo de estudio y que este dossier plasma una imagen sustantiva de lo acontecido y con un signo singular sobre y para Latinoamérica. 


\section{Andrea Gutiérrez / angut2@gmail.com}

Licenciada y Doctora en Geografía de la Universidad de Buenos Aires (UBA). Investigadora CONICET y Profesora Asociada del Departamento de Geografía (UBA). Directora del Programa Transporte y Territorio, y de la Revista Transporte y Territorio (Instituto de Geografía, UBA).

\section{Jorge Blanco / jblancodis@gmail.com}

Profesor en Geografía y Magister en Políticas Ambientales y Territoriales por la Universidad de Buenos Aires. Director del Instituto de Geografía y Profesor Asociado del Departamento de Geografía (UBA). 